\title{
Studies of Non-adiabatic Effects on Radial Pulsations in the Atmospheres of Rapidly Oscillating Ap Stars
}

\author{
R. Medupe, D. W. Kurtz \\ Department of Astronomy, University of Cape Town, Rondebosch, 7700, \\ South Africa \\ J. Christensen-Dalsgaard \\ Institute of Physics and Astronomy, Arhus University, DK-8000 C, \\ Denmark
}

\begin{abstract}
A code for solving non-adiabatic radial pulsation equations with consistent treatment of radiative transfer is used to study the oscillations in the atmospheres of the rapidly oscillating Ap stars. We find that our results are consistent with the presence of an observable node in the temperature eigenfunction.
\end{abstract}

\section{Introduction}

We discuss the behaviour of radial oscillations in the roAp stars. The discussion is based on the results of the solution of non-adiabatic radial pulsation equations with full treatment of radiative transfer, as described by Christensen-Dalsgaard \& Frandsen (1983). Our results can also be applied to nonradial oscillations, since it can be shown that the horizontal components of the motion are much smaller than the radial ones. We find that for roAp star pulsation frequencies, the ratio of horizontal to vertical displacement is of the order of $10^{-4}$.

\section{Results}

We report results based on an ATLAS9 model atmosphere with effective temperature $T_{\text {eff }}=7900 \mathrm{~K}$, and $\log g=4.2, g$ being surface gravity. A frequency of $2.44 \mathrm{mHz}$ is chosen, characteristic of the roAp stars. We find:

1. As expected, the solution is adiabatic high in the atmosphere and at large optical depths. In the photosphere, where the temperature $T$ is close to $T_{\text {eff }}$, the solution departs markedly from adiabaticity.

2. There is a significant variation with depth of temperature perturbations in the atmosphere for roAp frequencies. In particular there is a steep gradient in the relative Lagrangian temperature perturbation $\delta T / T$ in the photosphere (see Fig. 1). This is consistent with the semi-empirical results of Medupe \& Kurtz (1998), where they explained the observed rapid decline of photometric amplitudes with wavelength, as indicative of 
the steepness in $\delta T / T$. What remains to be seen is whether this behaviour of the temperature eigenfunction can also explain the observed pulsation phase variation with wavelength. Another point evident from Fig. 1 is that the variations occur over distances as short as about $100 \mathrm{~km}$.
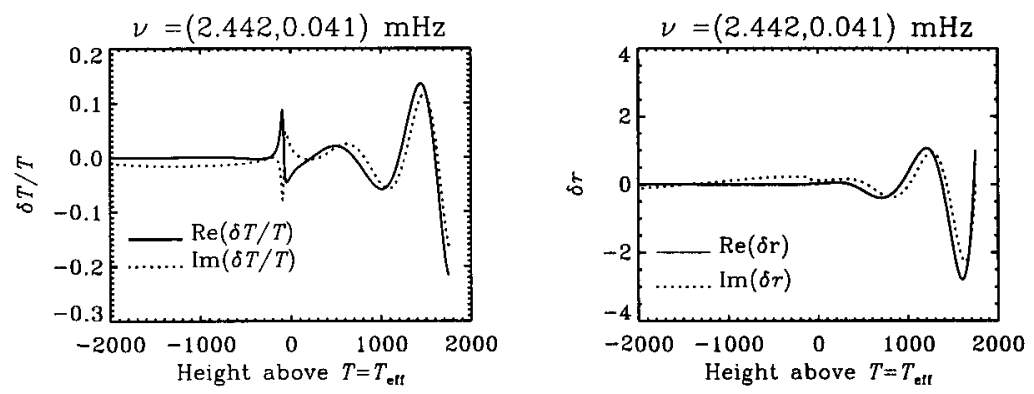

Figure 1. Temperature and displacement eigenfunctions.

3. The displacement eigenfunction $\delta r$ does not vary much near $T=T_{\text {eff }}$ but has a few nodes above the photosphere. Because we do not know yet the depth of formation of the $\mathrm{H} \alpha$ in $\alpha$ Cir, we are unable to determine with certainty which of the nodes in the displacement correspond to the node in the bisector velocities of the $\mathrm{H} \alpha$ as reported by Baldry et al. (1998).

4. Because of the second point, parameters such as

$$
f=\left|\frac{\delta T / T}{\delta R / R}\right| \text { and } C=\left|\frac{\delta P / P}{\delta R / R}\right|
$$

( $R$ being the photospheric radius), that are often used in analytical formulae for mode-identification and assumed to be constant, need to be properly determined because they are depth dependent in roAp stars.

\section{References}

Baldry, I. K., Bedding, T. R., Viskum M., Kjeldsen, H., \& Frandsen, S. 1998, MNRAS, 295, 33

Christensen-Dalsgaard, J. \& Frandsen, S. 1983, Solar Phys., 82, 165

Medupe, R. \& Kurtz, D. W. 1998, MNRAS, 299, 371 